\title{
Exercise electrocardiogram and single vessel coronary artery disease
}

\author{
Anoop Chauhan, Suren I Thuraisingham, DL Stone
}

\begin{abstract}
Summary
The aim of this study was to assess the efficiency of exercise tests in identifying ischaemia in the territories supplied by the three main coronary arteries. We prospectively analysed 578 patients with single vessel coronary disease $(>50 \%$ stenosis in one vessel and completely normal other vessels). Patients with single vessel coronary artery disease were divided into three groups: patients with significant left anterior coronary artery disease (group 1, $n=234$ ); patients with significant right coronary artery disease (group 2, $n=201$ ); patients with significant left circumflex disease (group 3, $n=143$ ). Our study, which is the largest prospective study of patients with angiographically documented single vessel coronary artery disease, suggests that the exercise electrocardiogram is a poor predictor of circumflex coronary artery ischaemia. In addition, the site of ST depression identified from the electrocardiogram was a poor predictor of the site of myocardial ischaemia. No single lead could distinguish between the three groups and the location of coronary stenosis could not be predicted by location of ST depression.
\end{abstract}

Keywords: exercise electrocardiogram, coronary artery disease

Exercise electrocardiography has now established itself as a major tool for the assessment of patients with suspected coronary artery disease. It has a sensitivity in the region of $68 \%$ (range $23-100 \%$ ) and a specificity of $77 \%$ (range $17-100 \%$ ) based on meta-analytical data. ${ }^{1}$ The aim of this study was to assess the sensitivity of this technique in identifying ischaemia occurring in the presence of significant coronary stenosis in individual coronary arteries.

Regional Cardiac

Unit, Papworth

Hospital, Papworth

Everard, Cambridge, UK

A Chauhan

SI Thuraisingham

Correspondence to Dr A Chauhan, Department of Cardiology, Blackpool Victoria Hospital, Whinney Heys Road, Blackpool FY3 8NR, UK

Accepted 29 January 1997

We prospectively analysed 578 patients with significant single vessel coronary disease who were investigated at our institution during the period of 1 October 1990 to 30 September 1993. Significant coronary artery disease was defined as $>50 \%$ stenosis in one coronary artery and normal other coronary arteries. Patients were divided into three groups: patients with significant left anterior descend- ing coronary artery disease (LAD group); patients with significant right coronary artery disease (RCA group); and patients with significant left circumflex disease (LCX group). Patients with $Q$ waves on the electrocardiogram (ECG), bundle branch block, interventricular conduction defect, left ventricular hypertrophy, associated valvular heart disease, or hypertrophic cardiomyopathy were excluded from the study. Exercise tests were performed using the standard Bruce Protocol prior to angiography in all patients (boxes $1 \& 2$ ). Three ECG leads were continuously monitored (V3, V5, and aVF) and 12-lead ECGs were obtained every three minutes and at peak exercise. The exercise ECG data were evaluated for the presence of exercise-induced ST depression by two independent, blinded investigators. The exercise test was said to be positive if there were $>1 \mathrm{~mm} \mathrm{ST}$ segment depression $80 \mathrm{~ms}$ after the J point.

\begin{tabular}{|c|c|c|c|}
\hline \multicolumn{4}{|c|}{ Bruce protocol } \\
\hline \multicolumn{4}{|c|}{$\begin{array}{l}\text { - multistage treadmill testing } \\
\text { - continuous exercise with } 2 \% \text { increments at } \\
3 \text { minutes: }\end{array}$} \\
\hline Stage & $\begin{array}{l}\text { Speed } \\
(m p h)\end{array}$ & $\begin{array}{c}\text { Grade } \\
(\%)\end{array}$ & $\begin{array}{l}\text { Total duration } \\
\text { (min) }\end{array}$ \\
\hline I & 1.7 & 10 & 3 \\
\hline II & 2.5 & 12 & 6 \\
\hline III & 3.4 & 14 & 9 \\
\hline IV & 4.2 & 16 & 12 \\
\hline V & 5.5 & 20 & 15 \\
\hline VI & 6.0 & 22 & 18 \\
\hline
\end{tabular}

Box 1

Bruce protocol: features suggestive of ischaemia

- chest pain typical of angina pectoris induced or increased by exercise

- significant drop $(>10 \mathrm{mmHg})$ in systolic blood pressure during exercise measured from the peak pressure reached during exercise or progressive fall or drop in systolic blood pressure persistently below baseline despite an increase in workload

- the occurrence of serious arrhythmias (eg, high grade ventricular arrhythmias)

- horizontal or downsloping ST segment depression of $0.1 \mathrm{mV}$ or more at $80 \mathrm{~ms}$ after the $\mathrm{J}$ point in three consecutive beats

- ST segment elevation in a lead without a Qwave 


\section{Results}

The table shows the patient variables and exercise test results. Statistical analysis was performed using the analysis of variance and the chi-square test when appropriate. A p-value of $<0.05$ was considered statistically significant. The sensitivity of the test was defined as the percentage of patients who had a positive exercise test. There were no significant differences between the three groups in age, sex, exercise duration, exercise heart rate and systolic pressure. The sensitivity of the test was similar in the LAD and RCA groups but was significantly lower in the LCX group. Overall, $340(58.8 \%)$ out of 578 patients studied had a positive exercise test.

\section{Discussion}

This is the largest prospective study of patients with angiographically documented single vessel coronary artery disease. Previous studies have either included patients with multivessel disease and have not excluded patients with electrocardiographic evidence of previous myocardial infarction or the numbers studied have been small. ${ }^{2-4}$ Our results suggest that exercise ECG is a poor predictor of circumflex coronary artery ischaemia. The electrocardiographic site of ST depression is also not a good predictor of the site of myocardial ischaemia. No single lead could distinguish between the three groups and the location of coronary artery stenosis could not be predicted by location of ST depression. This is in accord with some studies ${ }^{3-4}$ but is at variance with others. ${ }^{5}$ Our study, however, is the largest series reported and further highlights the value and limitations of the exercise ECG in localising coronary artery disease.

It is not surprising that the site of ST segment changes does not identify the location of coronary artery disease accurately as a number of important variables can influence the results. The amount of myocardium supplied by each coronary artery varies in individuals and is further influenced by proximal or distal obstruction. The severity of stenosis, variations in the extent of collateral flow, as well as variations in lead positioning may also be important. However, it has been shown previously that lead positions are not related to sensitivity or specificity of disease and standard anti-anginal drugs (beta-blockers, calcium antagonists, and nitrates) also do not significantly affect the sensitivity and specificity of ST segment depression. ${ }^{1}$

ST segment depression alone has a lower correlation than the occurrence of $\mathrm{Q}$-waves on the ECG in identifying the site of coronary disease. ${ }^{4}$ This is due to the multiple mechanisms by which ST segment depression can be produced. Subendocardial ischaemia produces ST depression in leads overlying the area whereas transmural ischaemia can produce ST depression in distant 'reciprocal leads'. Under these circumstances no ECG criteria

\section{Summary/learning points}

- exercise electrocardiography has a sensitivity in the region of $68 \%$ (range $23-100 \%$ ) and a specificity of $77 \%$ (range $17-100 \%$ )

- the exercise ECG is a poor predictor of circumflex coronary artery ischaemia

- the electrocardiographic site of ST depression is also not a good predictor of the site of myocardial ischaemia

\section{Box 3}

Table Patient variables and exercise test results

\begin{tabular}{llll}
\hline & $\begin{array}{l}L A D \\
\text { group }\end{array}$ & $\begin{array}{l}\text { RCA } \\
\text { group }\end{array}$ & $\begin{array}{l}L C X \\
\text { group }\end{array}$ \\
\hline Total number & 234 & 201 & 143 \\
Age & $56 \pm 11$ & $58 \pm 13$ & $55 \pm 9$ \\
Male: female & $176: 47$ & $153: 48$ & $113: 30$ \\
$\begin{array}{l}\text { Exercise heart rate } \\
\quad \text { (beats/min) }\end{array}$ & $149 \pm 19$ & $153 \pm 23$ & $147 \pm 29$ \\
$\begin{array}{l}\text { Exercise systolic } \\
\quad \text { pressure (mmHg) }\end{array}$ & $172 \pm 30$ & $169 \pm 27$ & $166 \pm 29$ \\
$\begin{array}{l}\text { Exercise duration } \\
\quad \text { (min) }\end{array}$ & $7.3 \pm 3.1$ & $7.6 \pm 2.8$ & $7.5 \pm 3.3$ \\
$\begin{array}{l}\text { Positive ET } \\
\text { Sensitivity }\end{array}$ & 160 & 132 & 48 \\
& $68.4^{\star}$ & $65.7^{\star}$ & 33.6
\end{tabular}

Location of ST depression

(number (\% of positive tests))

Lead I

II

AVL

AVF

V1

$\mathrm{V} 2$

V3

$\mathrm{V} 4$

V5

V6

$\begin{array}{ccc}9(6) & 6(5) & 1(2) \\ 68(43) & 60(45) & 18(38) \\ 40(25) & 35(27) & 15(31) \\ 7(4) & 4(3) & 4(8) \\ 63(39) & 47(36) & 15(31) \\ 17(11) & 12(9) & 4(8) \\ 19(12) & 14(11) & 4(8) \\ 43(27) & 29(22) & 11(23) \\ 69(43) & 46(35) & 18(38) \\ 14(71) & 84(64) & 30(63) \\ 96(60) & 74(56) & 30(63)\end{array}$

Values are expressed as mean \pm SD where appropriate. $E T$, exercise test; LAD, left anterior descending coronary artery; LCX, left circumflex coronary artery; RCA, right coronary artery, ${ }^{\star} \mathrm{p}<0.01$ as compared to the LCX group.

distinguish between right coronary and circumflex coronary artery disease, even in right dominant circulation. ${ }^{4}$

\section{Conclusion}

Our study has demonstrated that the exercise ECG is a poor predictor of circumflex coronary artery ischaemia. The electrocardiographic site of ST depression is also not a good predictor of the site of myocardial ischaemia. Although the exercise ECG remains a major tool in the investigation of patients with coronary artery disease, our study highlights the limitations of this technique in localising coronary artery disease. In the assessment of patients with suspected or known single vessel disease the combination of other investigational modalities such as radio-isotope myocardial perfusion scanning and stress echocardiography may provide additional important information. 
1 Detrano R, Gianrossi R, Froelicher V. The diagnostic accuracy of the exercise electrocardiogram: a meta-analysis of 22 years of research. Prog Cardiovasc Dis 1989; 32: 173 206.

2 Fox RM, Hakki A, Iskandrian AS. Relation between electrocardiographic and scintigraphic location of myocardial ischemia during exercise in one-vessel coronary artery disease. Am 7 Cardiol 1984; 53: 1529-31.

3 Dunn RF, Freedman B, Bailey IK, Uren RF, Kelly DT.

Dunn RF, Freedman B, Bailey IK, Uren RF, Kelly DT.
Location of coronary artery disease with exercise electroLocation of coronary artery disease with exercise electro-
cardiography: correlation with thallium-201 myocardial perfusion scanning. Am $\mathcal{f}$ Cardiol 1981; 48: 837-43.
4 Fuchs RM, Achuff SC, Grunwald L, Yin F, Griffith L. Electrocardiographic localization of coronary artery narrowings: studies during myocardial ischemia and infarction in patients with one-vessel disease. Circulation 1982; 66: $1168-76$.

5 Fox KM, Selwyn A, Oakley D, Shillingford JP. Relation between the precordial projection of ST segment changes after exercise and coronary angiographic findings. $A m \mathcal{F}$ Cardiol 1979; 44: 1068-79.

\title{
Warfarin potentiation with bezafibrate
}

\author{
Timothy RO Beringer
}

\author{
Summary \\ Two cases are described in which lack of \\ awareness of the potentiation of bezafi- \\ brate on warfarin was of clinical impor- \\ tance.
}

Keywords: bezafibrate, warfarin

Short- and long-term anticoagulant medication is widely and increasingly prescribed in a growing elderly population for the prevention and treatment of venous and arterial thromboembolism. Many of these patients have atherosclerosis and are also on lipid-lowering treatments. Increasing age and multiple medications are predisposing factors to adverse drug reactions. ${ }^{1}$ In addition, the list of drugs which may interact with warfarin continues to grow, with recent additions including the 4quinolone antibiotic ciprofloxacin, the selective serotonin re-uptake inhibitor group of antidepressants, and omeprazole.

\section{Case reports}

Case 1

A 65-year-old man presented with a fourday history of increasing weakness in the left arm and leg which had markedly worsened on the day of admission. He provided a history of childhood rheumatic fever, myocardial infarctions at the age of 53 and 58 years, and had undergone St Jude mitral valve replacement and coronary artery bypass grafting at the age of 64 years. $\mathrm{He}$ had subsequently received satisfactory therapeutic long-term anticoagulation with warfarin 4-5 mg daily. He was also receiving lipid-lowering therapy with bezafibrate $400 \mathrm{mg}$ daily, frusemide $40 \mathrm{mg}$, and amiloride hydrochloride $5 \mathrm{mg}$ daily.

Clinical examination confirmed the presence of left visual inattention, left upper motor neuron seventh nerve weakness and grade 4 power in the left arm and leg with preserved sensation. Computed tomography (CT) scan of brain demonstrated an area of reduced attenuation in the right frontal parietal region in keeping with infarction. His interna- tional normalised ratio (INR) was sub-therapeutic at 1.5 (reference range 2.0-3.5), blood urea was $8.2 \mathrm{mmol} / 1$ (reference range $3.3-8.8$ ) and serum creatinine $95 \mu \mathrm{mol} / 1$ (reference range 40-110). He was commenced on intravenous heparin $30000 \mathrm{IU}$ over 24 hours and his warfarin was continued. His INR failed to rise to therapeutic levels on $6 \mathrm{mg}$ rising to $7 \mathrm{mg}$ warfarin daily. Review of his medication revealed a low dose of bezafibrate of $200 \mathrm{mg}$ daily and institution of his previous dose of $400 \mathrm{mg}$ daily resulted in a therapeutic INR of 2.5 within 48 hours and withdrawal of his heparin. His limb weakness and visual inattention recovered, and he returned home walking independently. The patient then volunteered that during the week prior to admission he had not taken his normal dose of bezafibrate. He was unaware of the interaction between bezafibrate and warfarin. It is probable that the inadvertent reduction of bezafibrate had contributed to sub-therapeutic anticoagulation and the development of an embolic cerebrovascular event from a cardiac source.

Case 2

A 75-year-old woman was admitted after a fall resulting in a fracture of her right surgical neck of humerus and right neck of femur. She had a previous history of hypertension, heart failure, peripheral vascular disease, chronic obstructive airways disease and had been on treatment with warfarin from the age of 60 years following occlusion of a femoral-popliteal bypass. She had been commenced on lipidlowering therapy with bezafibrate $400 \mathrm{mg}$ daily in addition to a low-fat diet at the age of 69 years. Additional medication on admission included clonidine $100 \mu \mathrm{g}$ daily, amiloride $5 \mathrm{mg}$ daily, frusemide $40 \mathrm{mg}$ daily, salbutamol and ipratropium bromide inhalers, and her normal therapeutic dose of warfarin $1 \mathrm{mg}$ daily.

Initial investigations included a haemoglobin of $9.0 \mathrm{~g} / \mathrm{dl}$, urea of $9.7 \mathrm{mmol} / \mathrm{l}$, creatinine of $114 \mu \mathrm{mol} / 1$ and INR of 5.29. Her INR fell to a sub-therapeutic value of 1.2 five days following withdrawal of her oral warfarin. Following delayed surgery her warfarin was re-com- 